\title{
'||||||||||||||||||||||||||||||||||||||||||||||||||||||||||||||||||||.
}

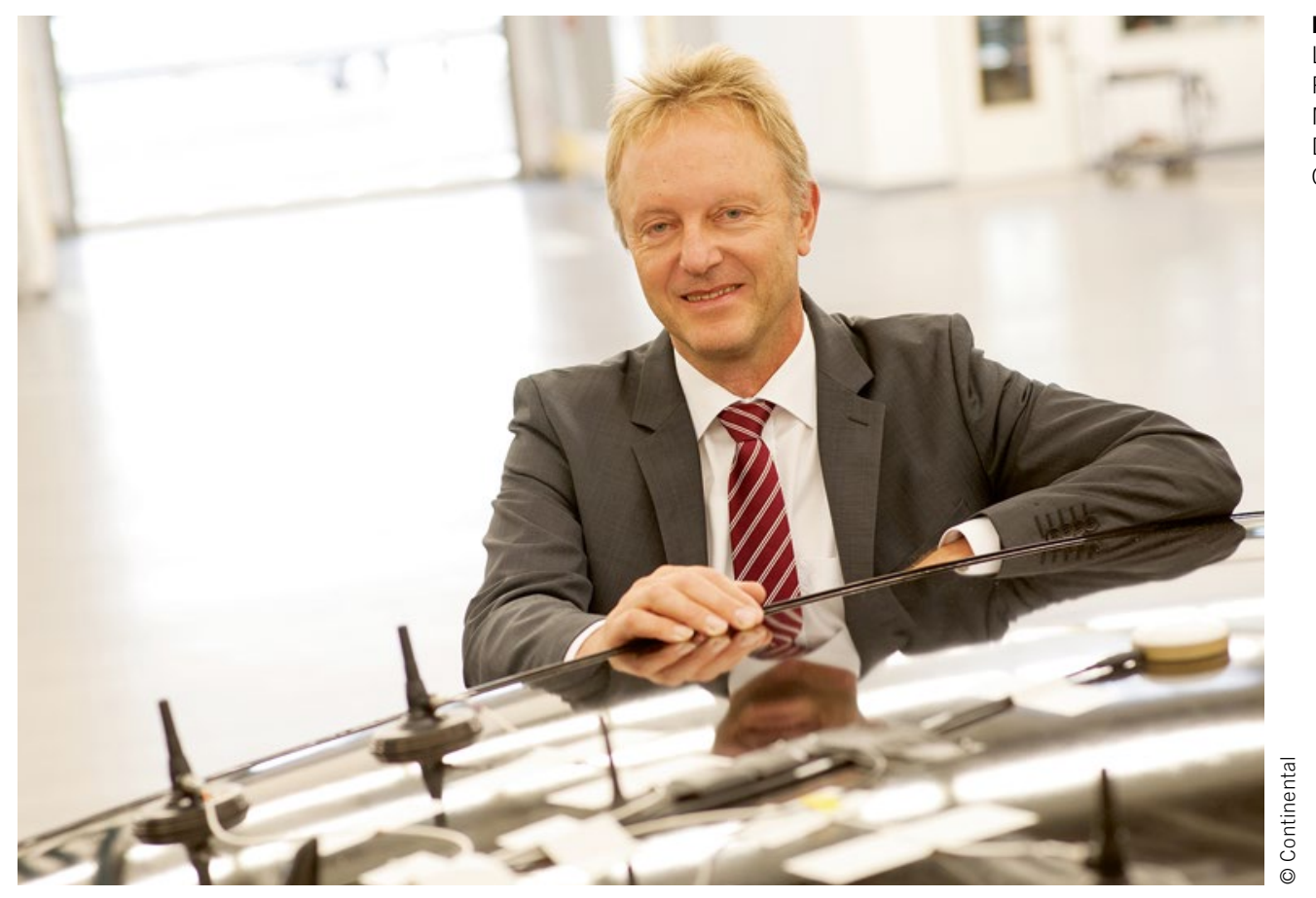

Dr.-Ing. Bernhard Klumpp Leiter Geschäftsbereich Passive Sicherheit und Sensorik, Mitglied der Geschäftsleitung, Division Chassis \& Safety,

Continental

\section{Sensoren sind unsere Beschützer im Notfall}

Nach Jahrzehnten der Reduktion stieg die Anzahl der Verkehrstoten in 2015 in Deutschland wieder leicht an. Um dieser Entwicklung entgegenzuwirken, ist die Verbindung der passiven Systeme mit denen der aktiven Sicherheit von großer Bedeutung. Und durch die Vernetzung verschiedener Sensorinformationen zu einem „intelligenten“ und ganzheitlichen Sicherheitskonzept wird die Fahr(zeug)sicherheit weiter verbessert. Das dadurch entstehende integrale Sicherheitssystem hilft dabei, zum einen die Insassen und zum anderen schwächere Verkehrsteilnehmer besser zu schützen. So kommen wir bei Continental der Vision vom unfallfreien Fahren Stück für Stück näher.

Was bedeutet dies konkret? Auch der Bereich der integralen Sicherheit unterliegt - wie andere Bereiche - einem stetigen Wandel. Sensoren und Systeme der aktiven, unfallvermeidenden Sicherheit werden mit den Komponenten der passiven, unfallfolgenabmildernden Sicherheitstechnik verbunden. Ziel ist es, durch diese Vernetzung mehr Informationen und somit ein genaueres Bild über das Fahrzeugumfeld und die Gefahrensituation zu erhalten. Sensoren bilden die Basis als Informationslieferanten, „intelligente“ Steuergeräte bewerten diese (Daten) und entscheiden über die Auslösung von möglichen Sicherheitsmaßnahmen. Sie sind somit unsere Beschützer im Notfall. Unsere Continental-Ingenieure entwickeln immer „intelligenter“ werdende Systeme, aus denen ein ganzheitliches Sicherheitskonzept entsteht.
Kommt es dennoch zu einem Unfall, gilt es, die Unfallfolgen zu verringern, zum Beispiel mit der Post-Crash-Braking-Funktion. Dabei sorgen Systeme dafür, das Fahrzeug nach einem ersten Anprall schnell und sicher zum Stehen zu bringen, ohne dass es dabei in einen weiteren Unfall verwickelt wird. Abhängig von der Schwere des Unfalls werden parallel dazu Notrufe ausgelöst. Bei Schleuderunfällen werden die Informationen der Fahrdynamiksensoren in den Algorithmus der Airbagauslösung einbezogen und beispielsweise mit den Bildern einer verfügbaren FAS-Kamera kombiniert. In einem idealen Ablauf werden die Airbags kaskadiert ausgelöst. So kann ein größtmöglicher Schutz der Insassen sichergestellt werden.

Die Vernetzung der Sensorinformationen findet nicht nur innerhalb des Fahrzeugs statt. Auch fahrzeugübergreifend werden sicherheitsrelevante Informationen ausgetauscht, um nachfolgende oder entgegenkommende Verkehrsteilnehmer zu warnen - inklusive der schwächeren Verkehrsteilnehmer. Zu den vorausschauenden Systemen zählt die Fahrzeug-zu-X-Kommunikationstechnik. Dabei übermitteln Fahrzeuge untereinander und zur Infrastruktur sicherheitsrelevante Informationen, um mögliche kritische Verkehrssituationen frühzeitig zu erkennen.

Nur durch eine verstärkte Vernetzung der Systeme kann die Sicherheit im Straßenverkehr erhöht werden. Der sichere Austausch von Informationen ist dabei von großer Bedeutung. Ganzheitliche Sicherheitskonzepte machen es möglich, die Anzahl der Überlebenden wieder zu erhöhen. 